\title{
Toppling dynamics of regularly spaced dominoes in an array
}

\author{
Tengfei Shi ${ }^{1}$, Yang Liu ${ }^{2}$, Nannan Wang ${ }^{1}$, Caishan Liu ${ }^{1 *}$ \\ ${ }^{1}$ State Key Laboratory for Turbulence and Complex Systems, College of Engineering \\ Peking University. Beijing, China 100871; \\ ${ }^{2}$ College of Engineering, Mathematics and Physical Sciences \\ University of Exeter, North Park Road, Exeter, UK EX4 4QF;
}

\begin{abstract}
This paper studies a new comprehensive model for toppling dynamics of regularly spaced dominoes in an array. The model has unlocked the hypotheses introduced by Stronge and Shu [1], which can provide us some essential insights into the mechanism of domino wave. Extensive comparisons are made between the proposed model and the experimental results studied in existing literature. Our numerical studies show that the existing theoretical models are special cases of the proposed model, and the fluctuation in the waveform of propagation speed observed from experiments was caused by the irregular multiple impacts between colliding dominoes. Influence of physical parameters of domino on the natural speed of toppling dominoes is also considered, and it is found that the coefficients of friction and restitution between colliding dominoes have more effects due to the energy dissipation during impact.
\end{abstract}

\section{Introduction}

Domino effect is a special word commonly used to describe the destabilization of marginally stable systems in nature, such as the phenomena of earthquake rupture [2, 3], signal propagation within a neural network [4,5], as well as the wave behaviour triggered in nanomaterials [6]. It is also used to describe the accidents appearing in process industry [7], chemical reaction [8], and sometimes is even used in sociology and economics [9,10]. From the view point of mechanics, manifestation of the domino phenomenon can be physically implemented via successive destabilization of neighbouring dominoes in a regularly spaced array. Since each domino in the array is marginally stable, destabilizing force can propagate through the array in a wave of destabilizing collisions once it is sufficient to topple the first domino. Modeling the dynamics of such destabilization is a topic of ongoing research, see e.g., [11, 12, 13, 14]. The existing works have focused on investigating the characteristics of the domino wave, such as the necessary conditions for toppling and the natural speed of wave propagation (see e.g., [1]). However, excessive hypotheses have been introduced in these studies which undermine the validity of the model.

This paper studies a new model for domino system

\footnotetext{
*Address all correspondence to this author: liucs@pku.edu.cn.
}

by unlocking several hypotheses proposed in the Stronge's model [1]. A detailed analysis of domino's dynamical response is performed with consideration for the complex interactions among a group of neighboring dominoes. These interactions include various discrete events, such as the stick-slip motion caused by friction, the separation and impact between two dominoes, and the detachment followed by the impact between the domino and the ground. Modeling such nonsmooth dynamics has been a challenging research work. Daykin [9] for the first time raised the question of how fast dominoes fall. McLachlan et al. [15] experimentally studied this problem and found a scaling law for its wave speed providing that the dominoes were equally spaced with zero thickness in a straight line. With these assumptions, a functional relation between the wave speed and the relative spacing (i.e. the ratio of domino spacing and height) of dominoes was identified. Later on, this functional relation was concreted by Bert [16] then by Efthimiou et al. [17]. Nevertheless, these theoretical analyses were established by introducing excess assumptions, leading to the solutions with large errors in comparison with existing experimental results [1, 18, 15, 19]. Stronge pioneered the physical-based studies on the domino wave by performing considerable realistic analyses under two limiting conditions. In [18], he presented theoretical results for the wave propagation assuming that there was only a single collision between neighbouring dominoes. Furthermore, Stronge and Shu [1] considered the domino wave triggered by a cooperative group with a large number of dominoes, where each domino leaned against a neighbor keeps contacting during toppling process. While the Stronge's model has fundamentally provided some insights into the mechanism of the domino wave, large discrepancies between his theoretical results and associated experimental data still exist. This suggests that a more accurate and general model is needed for precisely characterizing the domino wave.

In order to obtain accurate theoretical results, this work concerns a computational method for numerical simulation of the domino system. When there are strong couplings between frictions and collisions at several simultaneous contact points, adoption of a proper collision law for precise numerical simulation of multiple impacts is crucial. Shaw 
[20] has proposed a numerical model for the domino system under the assumptions that the collisions between dominoes are inelastic and the angular momentums during collisions are conserved. Similar assumptions were used by van Leeuwen [13] and Fujii et. al [21] who developed a numerical model with consideration of friction effect. Both works conclude that it is difficult to interpret experimental results, since the collision laws used in these two domino models are too simplistic to precisely capture the complex motion of falling dominoes. Using the constitutive law involving the contact force and deformation of the collision body is another way of quantifying impact interactions. For example, $\mathrm{Lu}$ et al. [14] has adopted the discrete element method to model the interactions between dominoes and the ground which shows a good agreement between numerical results and experimental observations. However, simulations using this method may encounter various difficulties in determination of physical parameters of the model or numerical integration of differential equations using small integration step. In [22, 23], Liu et al. proposed a numerical algorithm for multiple impacts between the bodies composed of rateindependent materials. The principle of this method is that the interface behaviours due to impacts can be described by using the relationship between the contact force and the elastic potential energy transferred from the kinetic energy of impacting bodies. Energy dissipation is quantified using an energetic coefficient, and the coupling effects among simultaneous contact points are reflected using the ratio of potential energy at each contact point. Moreover, numerical integration can be performed at the level of impulse in order to avoid using small variables, such as the time of impact and the length of local deformation. The multiple impacts model will be used in this paper to solve the complex impacts involved in the dynamics of the domino system.

The rest of the paper is organized as follows. In Section 2 , the dynamics of toppling dominoes and the multiple impacts model are introduced. Section 3 provides extensive comparisons between the proposed domino model and the theoretical and experimental results studied in [1, 13, 18, 21]. Special attention is paid to reveal the mechanism of domino falling and the cause of fluctuation in the waveform of domino's propagation speed. In addition, the influence of physical parameters of domino on the natural speed of toppling dominoes is also studied. Finally, in Section 4, some conclusions are drawn.

\section{The model}

This work considers the toppling dynamics for an array of regularly spaced dominoes. This section will study both the kinematic and dynamic models of this domino system with a special attention paid to the introduction of the multiple impacts model proposed by Liu et al. [22,23].

\subsection{Kinematic model}

Consider $n$ slender dominoes with each block of the mass $m$, the height $h$, and the thickness $d$, which stand on the ground with equal spacing $s$ as depicted in Fig 1, We introduce an inertial coordinate frame $\mathcal{F}_{I}=\{O ; \mathbf{i j}\}$ with the axis $\mathbf{i}$ along the ground. The centre of mass of the $i$ th block $o_{i}$ is located at $\left(x_{i}, y_{i}\right)$ in the frame $\mathcal{F}_{I}$. The motion of the $i$ th block can be represented using $\mathbf{q}_{i}=\left(x_{i}, y_{i}, \theta_{i}\right)$, where $\theta_{i}$ is the angular displacement and its positive value is along the clockwise direction. The body-fixed coordinate frame $\mathcal{F}_{i}=\left\{O_{i} ; \mathbf{i}_{i} \mathbf{j}_{i}\right\}$ for the $i$ th block is considered, and its transformation to the inertial coordinate frame $\mathcal{F}_{I}$ is given as

$$
\left[\begin{array}{c}
\mathbf{i}_{i} \\
\mathbf{j}_{i}
\end{array}\right]=\left[\begin{array}{cc}
\cos \theta_{i} & -\sin \theta_{i} \\
\sin \theta_{i} & \cos \theta_{i}
\end{array}\right]\left[\begin{array}{l}
\mathbf{i} \\
\mathbf{j}
\end{array}\right] \equiv \mathbf{R}_{i}\left[\begin{array}{l}
\mathbf{i} \\
\mathbf{j}
\end{array}\right] .
$$

For an array of equally spaced dominoes with $n$ elements, its motion can be fully described by a set of generalized coordinate, $\mathbf{q}=\left(\mathbf{q}_{1}, \mathbf{q}_{2}, \cdots, \mathbf{q}_{n}\right)^{T} \in \mathbb{R}^{3 n}$. Noting that, if the $\kappa$ th block is stable on the ground, the rest blocks $n-\kappa$ will not be involved in the dynamics, we can define a time-varying dimension for $\mathbf{q}$ : If the $(\kappa-1)$ th block is falling down towards its stable neighbor $\kappa$, the generalized coordinates for the current configuration of the system can be denoted as $\mathbf{q} \in \mathbb{R}^{3 \kappa}$.

Point contact between two bodies means that the position of the contact point is commonly occupied by a pair of points on the contours of the two contacting bodies. Let us designate $\left\{A_{i}, A_{i}^{\prime}\right\}$ as the pair of points for the point contact between the $i$ th block and the ground, and $\left\{B_{i}, B_{i}^{\prime}\right\}$ as the pair of points for the point contact between the $i$ th block and the $(i+1)$ th block, where the symbols without prime correspond to the points on the $i$ th block, and the ones with prime denote the points on the colliding bodies. The points $A_{i}$ and $B_{i}$ are fixed point on the $i$ th block, so the position vectors of these points in frame $\mathcal{F}_{I}$ can be written as

$$
\left\{\begin{aligned}
\mathbf{r}_{O A_{i}}= & x_{A_{i}} \mathbf{i}+y_{A_{i}} \mathbf{j} \\
= & \left(x_{i}-\frac{h}{2} \sin \theta_{i}+\frac{d}{2} \cos \theta_{i}\right) \mathbf{i} \\
& +\left(y_{i}-\frac{h}{2} \cos \theta_{i}-\frac{d}{2} \sin \theta_{i}\right) \mathbf{j} \\
\mathbf{r}_{O B_{i}}= & x_{B_{i}} \mathbf{i}+y_{B_{i}} \mathbf{j} \\
= & \left(x_{i}+\frac{h}{2} \sin \theta_{i}+\frac{d}{2} \cos \theta_{i}\right) \mathbf{i} \\
& +\left(y_{i}+\frac{h}{2} \cos \theta_{i}-\frac{d}{2} \sin \theta_{i}\right) \mathbf{j} .
\end{aligned}\right.
$$

Nevertheless, the positions of points $A_{i}^{\prime}$ on the ground and $B_{i}^{\prime}$ on the $(i+1)$ th block change with the motion of ith block. In order to find the positions of points $A_{i}^{\prime}$ and $B_{i}^{\prime}$, we first denote $P$ as an arbitrary point on the ground and $Q$ as an arbitrary one on the left surface of the $(i+1)$ th block.

$$
\left\{\begin{array}{l}
\mathbf{r}_{O P}=\xi \mathbf{i}, \\
\mathbf{r}_{O_{i+1} Q}=-\frac{d}{2} \mathbf{i}_{i+1}+\zeta \mathbf{j}_{i+1},
\end{array}\right.
$$

where, $\xi$ and $\zeta$ are unknown parameters that should be determined according to the following contact conditions [24]

$$
\begin{cases}\left(\mathbf{r}_{O A_{i}}-\mathbf{r}_{O P}\right) \cdot \mathbf{i} & =0 \\ \left(\mathbf{r}_{O B_{i}}-\mathbf{r}_{O O_{i+1}}-\mathbf{r}_{O_{i+1} Q}\right) \cdot \mathbf{j}_{i+1} & =0\end{cases}
$$

By substituting the relationships between parameters $\xi$ and $\zeta$ into Eq.(3), and applying the transformation matrix in 


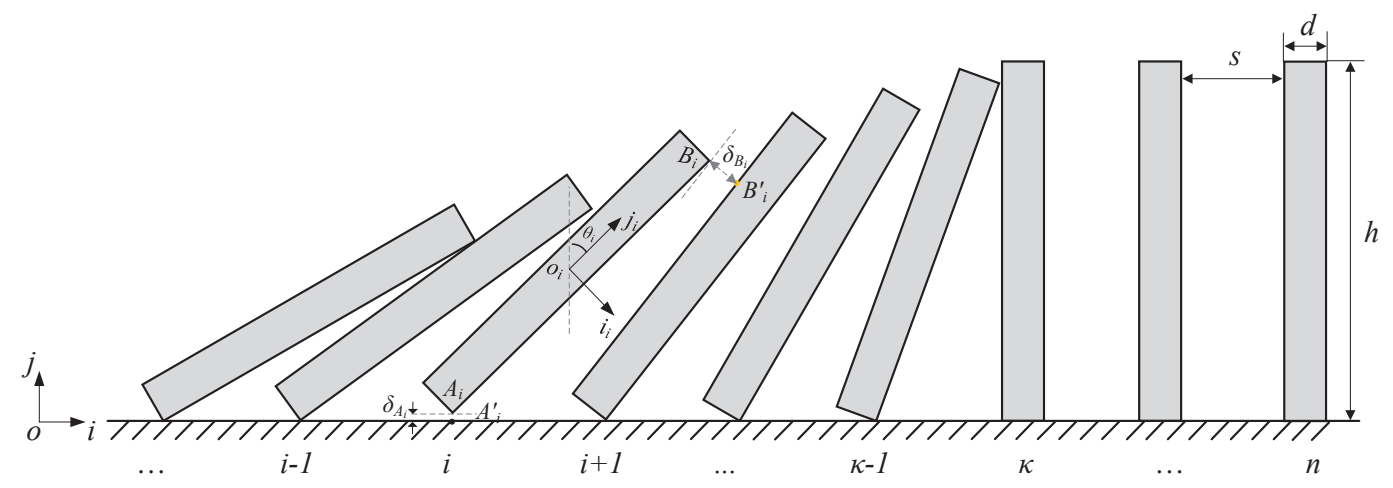

Fig. 1. Toppling dominoes on the ground.

Eq. (1), the position vectors for the points $A_{i}^{\prime}$ and $B_{i}^{\prime}$ in frame $\mathcal{F}_{I}$ can be obtained as

$$
\left\{\begin{aligned}
\mathbf{r}_{O A_{i}^{\prime}}= & x_{A_{i}} \mathbf{i}=\left(x_{i}-\frac{h}{2} \sin \theta_{i}+\frac{d}{2} \cos \theta_{i}\right) \mathbf{i}, \\
\mathbf{r}_{O B_{i}^{\prime}}= & x_{B_{i}^{\prime}} \mathbf{i}+y_{B^{\prime}} \mathbf{j} \\
= & {\left[x_{B_{i}} \cos ^{2} \theta_{i+1}+\left(x_{i+1}-\frac{d}{2} \cos \theta_{i+1}\right) \sin ^{2} \theta_{i+1}\right.} \\
& \left.+\left(y_{B_{i}}-y_{i+1}-\frac{d}{2} \sin \theta_{i+1}\right) \sin \theta_{i+1} \cos \theta_{i+1}\right] \mathbf{i} \\
& +\left[y_{B_{i}} \sin ^{2} \theta_{i+1}+\left(y_{i+1}+\frac{d}{2} \sin \theta_{i+1}\right) \cos ^{2} \theta_{i+1}\right. \\
& \left.+\left(x_{B_{i}}-x_{i+1}-\frac{d}{2} \cos \theta_{i+1}\right) \sin \theta_{i+1} \cos \theta_{i+1}\right] \mathbf{j} .
\end{aligned}\right.
$$

Let $\delta_{i}=\left(\delta_{A_{i}}, \delta_{B_{i}}\right)$ be the relative normal displacement of the potential contact set for the $i$ th domino block. They are defined as follows:

$$
\left\{\begin{aligned}
\delta_{A_{i}}= & \left(\mathbf{r}_{O A_{i}}-\mathbf{r}_{O A_{i}^{\prime}}\right) \cdot \mathbf{i}=y_{i}-\frac{h}{2} \cos \theta_{i}-\frac{d}{2} \sin \theta_{i}, \\
\delta_{B_{i}}= & \left(\mathbf{r}_{O B_{i}^{\prime}}-\mathbf{r}_{O B_{i}}\right) \cdot \mathbf{i}_{i+1} \\
= & \left(x_{i+1}-x_{i}\right) \cos \theta_{i+1}-\left(y_{i+1}-y_{i}\right) \sin \theta_{i+1} \\
& -\frac{d}{2}-\left(\frac{h}{2} \sin \theta_{i}+\frac{d}{2} \cos \theta_{i}\right) \cos \theta_{i+1} \\
& +\left(\frac{h}{2} \cos \theta_{i}-\frac{d}{2} \sin \theta_{i}\right) \sin \theta_{i+1},
\end{aligned}\right.
$$

where $\boldsymbol{\delta}_{\boldsymbol{i}} \geq 0$.

Taking time derivatives of Eqs. (2) and (5), the relative velocity of the two contact sets $\left\{A_{i}, A_{i}^{\prime}\right\}$ and $\left\{B_{i}, B_{i}^{\prime}\right\}$ can be expressed as

$$
\left\{\begin{array}{l}
\mathbf{v}_{A_{i}}^{r}=\mathbf{v}_{A_{i}}-\mathbf{v}_{A_{i}^{\prime}}=\frac{\mathrm{d} \mathbf{r}_{O A_{i}}}{\mathrm{~d} t}-\frac{\mathrm{d} \mathbf{r}_{O A_{i}^{\prime}}}{\mathrm{d} t}, \\
\mathbf{v}_{B_{i}}^{r}=\mathbf{v}_{B_{i}^{\prime}}-\mathbf{v}_{B_{i}}=\frac{\mathrm{d} \mathbf{r}_{O B_{i}^{\prime}}}{\mathrm{d} t}-\frac{\mathrm{d} \mathbf{r}_{O B_{i}}}{\mathrm{~d} t},
\end{array}\right.
$$

which can be decomposed along the normal and tangential directions of the contact surfaces as follows:

$$
\left\{\begin{array}{l}
\dot{\boldsymbol{\delta}}_{i}=\left[\begin{array}{c}
\dot{\delta}_{A_{i}} \\
\dot{\delta}_{B_{i}}
\end{array}\right]=\left[\begin{array}{c}
\mathbf{v}_{A_{i}}^{r} \cdot \mathbf{j} \\
\mathbf{v}_{B_{i}}^{r} \cdot \mathbf{i}_{i+1}
\end{array}\right]=\mathbf{w}_{i}^{T}\left(\mathbf{q}_{i}, \mathbf{q}_{i+1}\right)\left[\begin{array}{c}
\dot{\mathbf{q}}_{i} \\
\dot{\mathbf{q}}_{i+1}
\end{array}\right], \\
\boldsymbol{v}_{i}^{\tau}=\left[\begin{array}{c}
v_{A_{i}}^{\tau} \\
v_{B_{i}}^{\tau}
\end{array}\right]=\left[\begin{array}{c}
\mathbf{v}_{A_{i}}^{r} \cdot \mathbf{i} \\
\mathbf{v}_{B_{i}}^{r} \cdot \mathbf{j}_{i+1}
\end{array}\right]=\mathbf{n}_{i}^{T}\left(\mathbf{q}_{i}, \mathbf{q}_{i+1}\right)\left[\begin{array}{c}
\dot{\mathbf{q}}_{i} \\
\dot{\mathbf{q}}_{i+1}
\end{array}\right],
\end{array}\right.
$$

where $\dot{\mathbf{q}}_{i}=\left(\dot{x}_{i}, \dot{y}_{i}, \dot{\theta}_{i}\right)^{T}$ is the generalized velocity of the $i$ th block, and the matrixes $\mathbf{w}_{i}$ and $\mathbf{n}_{i}$ are given as

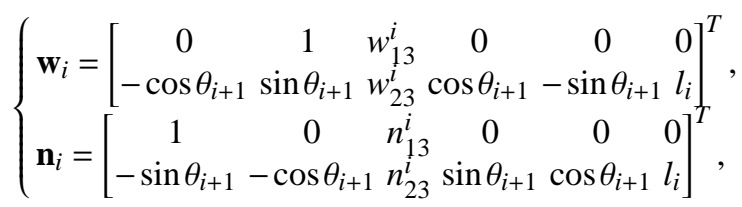

where

$$
\left\{\begin{aligned}
w_{13}^{i} & =\frac{h}{2} \sin \theta_{i}-\frac{d}{2} \cos \theta_{i} \\
n_{13}^{i} & =-\frac{h}{2} \cos \theta_{i}-\frac{d}{2} \sin \theta_{i} \\
w_{23}^{i} & =-\left(\frac{h}{2} \cos \theta_{i}-\frac{d}{2} \sin \theta_{i}\right) \cos \theta_{i+1} \\
& -\left(\frac{h}{2} \sin \theta_{i}+\frac{d}{2} \cos \theta_{i}\right) \sin \theta_{i+1}, \\
n_{23}^{i}=-\left(\frac{h}{2} \cos \theta_{i}-\frac{d}{2} \sin \theta_{i}\right) \sin \theta_{i+1} & \\
& +\left(\frac{h}{2} \sin \theta_{i}+\frac{d}{2} \cos \theta_{i}\right) \cos \theta_{i+1}, \\
l_{i}= & \frac{x_{B_{i}^{\prime}}-x_{i+1}+\frac{h}{2} \sin \theta_{i+1}+\frac{d}{2} \cos \theta_{i+1}}{\sin \theta_{i+1}}-\frac{h}{2} .
\end{aligned}\right.
$$

For the $\kappa$ th block, the bottom is kept line contact with the ground until it starts to fall. In order to simplify the interaction at this contact surface, we introduce $\lambda$ equidistant discrete contact points to replace the line contact as shown in Fig. 2, and the contact set for these points is written as $\left\{A_{\kappa p}, A_{\kappa p}^{\prime}\right\}$, where $p=1,2, \cdots, \lambda$. Here, we employ a new coordinate frame $\mathcal{F}_{K}=\left\{o_{K} ; \mathbf{i}_{K}^{\prime} \mathbf{j}_{K}^{\prime}\right\}$ located at the centre of bottom edge of the $\kappa$ th block. Then the position of the $p$ th point in the local coordinate frame can be express as $\left(x_{\kappa p}, 0\right)$, where $x_{\kappa p}=2 \frac{p-1}{\lambda-1} d-\frac{d}{2}$. Applying the transformation, the contact sets $A_{\kappa p}$ is given as

$$
\left\{\begin{array}{l}
\delta_{A_{k p}}=y_{\kappa}-\frac{h}{2} \cos \theta_{\kappa}-x_{\kappa p} \sin \theta_{\kappa} \equiv 0, \\
\dot{\delta}_{A_{\kappa p}}=\dot{y}_{K}+\left(\frac{h}{2} \sin \theta_{\kappa}-x_{\kappa p} \cos \theta_{\kappa}\right) \dot{\theta}_{\kappa}, \\
v_{A_{\kappa p}}^{\tau}=\dot{x}_{\kappa}-\left(\frac{h}{2} \cos \theta_{\kappa}+x_{\kappa p} \sin \theta_{\kappa}\right) \dot{\theta}_{\kappa},
\end{array}\right.
$$

where $\delta_{A_{\kappa p}}, \dot{\delta}_{A_{\kappa p}}$ and $v_{A_{\kappa p}}^{\tau}$ are the normal distance, the normal velocity, and the tangential velocity of the contact set $\left\{A_{\kappa p}, A_{\kappa p}^{\prime}\right\}$, respectively.

Considering $\kappa$ toppling blocks and the multi-points 


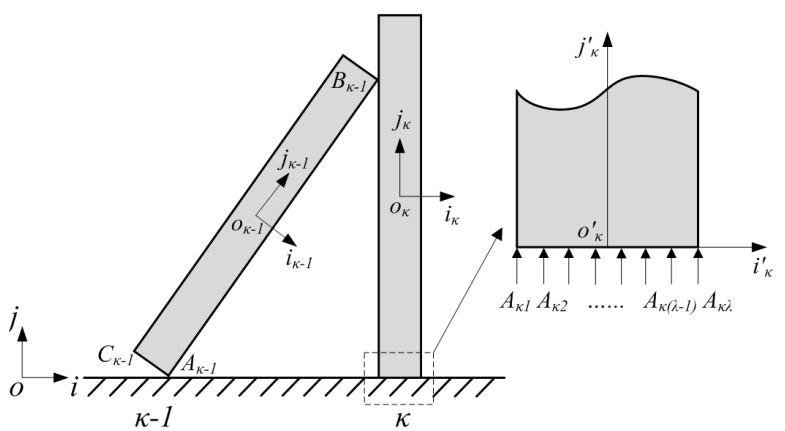

Fig. 2. Multi-points contact between the $\kappa$ th block and the ground.

contact at the bottom of the $\kappa$ th block, we have

$$
\left\{\begin{array}{l}
\boldsymbol{\delta}=\left(\boldsymbol{\delta}_{\mathbf{1}}, \boldsymbol{\delta}_{\mathbf{2}}, \cdots, \boldsymbol{\delta}_{\kappa-1}, \delta_{A_{k 1}}, \delta_{A_{\kappa 2}}, \cdots, \delta_{A_{\kappa \lambda}}\right)^{T} \in \mathbb{R}^{2 \kappa-2+\lambda} \\
\dot{\boldsymbol{\delta}}=\left(\dot{\boldsymbol{\delta}}_{\mathbf{1}}, \dot{\boldsymbol{\delta}}_{2}, \cdots, \dot{\boldsymbol{\delta}}_{\kappa-1}, \dot{\delta}_{A_{\kappa 1}}, \dot{\delta}_{A_{\kappa 2}}, \cdots, \dot{\delta}_{A_{\kappa \lambda}}\right)^{T} \in \mathbb{R}^{2 \kappa-2+\lambda} \\
\mathbf{v}^{\tau}=\left(\mathbf{v}_{1}^{\tau}, \mathbf{v}_{2}^{\tau}, \cdots, \mathbf{v}_{\kappa-1}^{\tau}, v_{A_{\kappa 1}}^{\tau}, v_{A_{\kappa 2}}^{\tau}, \cdots, v_{A_{\kappa \lambda}}^{\tau}\right)^{T} \in \mathbb{R}^{2 \kappa-2+\lambda}
\end{array}\right.
$$

Combining Eqs. (9) and (10), the components of the relative velocities at all of the contact points along their normal and tangential directions of the contact surfaces can be rewritten as

$$
\left\{\begin{array}{l}
\dot{\delta}=\mathbf{W}^{T} \dot{\mathbf{q}} \\
\mathbf{v}^{\tau}=\mathbf{N}^{T} \dot{\mathbf{q}}
\end{array}\right.
$$

where $\mathbf{W}$ and $\mathbf{N}$ are the Jacobian matrixes of the normal and the tangential constraints of the contact set, respectively. Taking time derivatives of Eq. (12), we can obtain the normal and the tangential relative accelerations

$$
\left\{\begin{array}{l}
\ddot{\boldsymbol{\delta}}=\mathbf{W}^{T} \ddot{\mathbf{q}}+\mathbf{S}^{\mathbf{n}}, \\
\dot{\mathbf{v}}^{\tau}=\mathbf{N}^{T} \ddot{\mathbf{q}}+\mathbf{S}^{\tau},
\end{array}\right.
$$

where $\mathbf{S}^{n}=\dot{\mathbf{W}}^{T} \dot{\mathbf{q}}$ and $\mathbf{S}^{\tau}=\dot{\mathbf{N}}^{T} \dot{\mathbf{q}}$.

\subsection{Dynamic model}

For $\kappa$ toppling blocks, the total kinetic energy is

$$
T(\mathbf{q}, \dot{\mathbf{q}})=\sum_{i=1}^{\kappa}\left(\frac{1}{2} m \dot{x}_{i}^{2}+\frac{1}{2} m \dot{y}_{i}^{2}+\frac{1}{2} I \dot{\theta}_{i}^{2}\right),
$$

where $I=\frac{1}{12}\left(h^{2}+d^{2}\right)$ is the moment of inertia of the block about its centre of mass. The gravitational potential energy of $\kappa$ blocks with respect to the ground can be expressed as

$$
U=\sum_{i=1}^{\kappa} m y_{i}
$$

In order to take the Coulomb's friction into account, we decompose the contact forces into the components along the normal and the tangential directions, which can be represented as

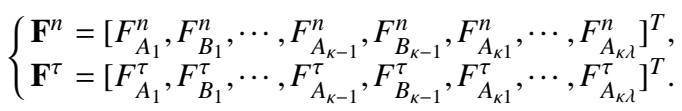

Now, defining a Lagrangian function $L=T-U$ to use the Euler-Lagrange equation, and considering the unilateral constraint at the potential contact set, we obtain the equations of motion for $\kappa$ toppling blocks

$$
\mathbf{M} \ddot{\mathbf{q}}=\mathbf{G}+\mathbf{W F}^{n}+\mathbf{N} \mathbf{F}^{\tau},
$$

where $\mathbf{M}=\operatorname{diag}(m, m, I, m, m, I, \cdots, m, m, I) \in \mathbb{R}^{3 \kappa \times 3 k}$ is the generalized mass matrix, $\mathbf{G}=[0,-m g, 0,0,-m g, 0, \cdots, 0-$ $m g, 0]^{T} \in \mathbb{R}^{3 k \times 1}$ is the generalized gravity, $g$ is the acceleration due to gravity.

In order to solve Eq. (17), the contact forces $\mathbf{F}^{n}$ and $\mathbf{F}^{\tau}$ need to be determined. The integrated linear complementary relationship studied in [25, 26] was adopted in this paper for detecting the contact state and for calculating the normal contact force at each contact set. To detect a contact state, the following two complementary conditions studied in [27,28] are considered,

$$
\left\{\begin{array}{l}
\delta_{i} \cdot F_{i}^{n}=0, \quad \delta_{i} \geq 0, F_{i}^{n} \geq 0, \\
\dot{\delta}_{i} \cdot F_{i}^{n}=0, \quad \dot{\delta}_{i} \geq 0, F_{i}^{n} \geq 0,
\end{array} \quad i=1, \cdots 2 \kappa-2+\lambda\right.
$$

It should be noted that contact is established only when both conditions $\delta_{i}=0$ and $\dot{\delta}_{i}=0$ are satisfied simultaneously. The normal contact force $F_{i}^{n}=0$ if $\delta_{i}>0$ or $\delta_{i}=0$ together with $\dot{\delta}_{i}>0$. When $\delta_{i}=0$ and $\dot{\delta}_{i}<0$, this contact point is involved in an impact state, which will be discussed later. To determine the contact force in a sustained contact process, the linear complementary relationship must be established at the acceleration level

$$
\ddot{\delta}_{i} \cdot F_{i}^{n}=0, \ddot{\delta}_{i} \geq 0, F_{i}^{n} \geq 0 .
$$

Considering the tangential contact force acting on the rough interface, we introduce the Coulomb friction into the contact set, so the tangential forces can be written as

$$
\begin{cases}F_{i}^{\tau}=-\mu_{i} F_{i}^{n} \frac{v_{i}^{\tau}}{\left|v_{i}^{\tau}\right|}, & \text { if } v_{i}^{\tau} \neq 0 \\ F_{i}^{\tau} \leq \mu_{i}^{s} F_{i}^{n}, & \text { if } v_{i}^{\tau}=0 .\end{cases}
$$

where $\mu_{i}>0$ and $\mu_{i}^{s}>\mu_{i}$ are the slip and the stick friction coefficients at the $i$ th contact set, respectively.

Combining the governing equation (17) with Eqs. (13), (19) and (20), the dynamics of the domino system out of collision events can be solved numerically.

\subsection{Impact dynamics}

To use the impact model proposed by Liu et al. [22,23], the following assumptions are made: (i) the displacement during impact is invariant, and (ii) the non-impulsive forces during impact can be neglected. Under such assumptions, multiplying both sides of Eq. (17) by $\mathrm{d} t$, the dynamic equations at the impulsive level can be written as

$$
\mathbf{M d} \dot{\mathbf{q}}=\mathbf{W d} \mathbf{P}^{n}+\mathbf{N d} \mathbf{P}^{\tau},
$$


where $\mathrm{d} \mathbf{P}^{n}=\boldsymbol{F}^{n} \mathrm{~d} t$ and $\mathrm{d} \mathbf{P}^{\tau}=\mathbf{F}^{\tau} \mathrm{d} t$ are the normal and the tangential infinitesimal impulses, respectively.

To obtain the velocity incremental d $\dot{\mathbf{q}}$, the distribution of infinitesimal impulses $\mathrm{d} \mathbf{P}^{n}$ and $\mathrm{d} \mathbf{P}^{\tau}$ at these contact sets should be determined. According to the impact model studied in [22], the distribution of normal impulse is related to the evolution of the potential energy between different contact sets, and the tangential impulse can be obtained by introducing the Coulomb friction law. So, the potential energy $E_{i}$ induced by the work $\mathrm{d} w_{i}$ done by the contact force through deformation between contact sets can be transformed to the velocity and impulsive level as

$$
\mathrm{d} E_{i}=-\mathrm{d} w_{i}=-F_{i}^{n} \mathrm{~d} \delta_{i}=-\dot{\delta}_{i} \mathrm{~d} P_{i}^{n},
$$

where $\mathrm{d} P_{i}^{n}$ is the infinitesimal impulse at the $i$ th contact set.

Define $\eta_{i}$ as the transition efficiency between the potential energy $\mathrm{d} E_{i}$ and the work $\mathrm{d} w_{i}$ done by the normal contact forces through deformation, it gives

$$
\mathrm{d} w_{i}=-\eta_{i} \mathrm{~d} E_{i}, \quad \eta_{i}\left(\dot{\delta}_{i}\right)= \begin{cases}1, & \text { when } \dot{\delta}_{i} \leq 0, \\ e_{i}^{2}, & \text { when } \dot{\delta}_{i}>0,\end{cases}
$$

where $\dot{\delta}_{i} \leq 0$ means the compression phase, $\dot{\delta}_{i}>0$ indicates the expansion phase, and $e_{i}$ is a macroscopic parameter for encapsulating the loss of energy induced by various factors to be confined in a single compression-expansion cycle. Here, we assume that no pre-press energy exists, i.e. $E_{i}(0)=0$ when $P_{i}^{n}=0$, so the potential energy corresponding to the impulse $P_{i}^{n}$ can be obtained by using Eqs. (22) and (23)

$$
E_{i}\left(P_{i}^{n}\right)=-\int_{0}^{P_{i}^{n}} \eta_{i} \dot{\delta}_{i} \mathrm{~d} P_{i}^{n}
$$

where $P_{i}^{n}$ is always positive and increases monotonically when the contact is retained.

Suppose that the relationship between the normal contact force and normal deformation satisfies a constitutive equation in a power form,

$$
F_{i}^{n}=f_{i}\left(\delta_{i}\right)=k_{i}\left(-\delta_{i}\right)^{\alpha_{i}}
$$

where $k_{i}$ is the contact stiffness, the negative sign indicates that $\left(-\delta_{i}\right)>0$ when contact holds, and $\alpha_{i}$ is set to 1.5 for a Hertzian model, and to 1.0 for a contact model with a linear constitutive relationship.

Now combining Eqs. (24) and (25), we have

$$
F_{i}^{n}=\left(\alpha_{i}+1\right)^{\frac{\alpha_{i}}{\left(\alpha_{i}+1\right)}} k_{i}^{\frac{\alpha_{i}}{\left(\alpha_{i}+1\right)}} E_{i}^{\frac{\alpha_{i}}{\left(\alpha_{i}+1\right)}} .
$$

For the detailed derivation of Eq. (26), one can refer to [26]. By using Eq. (26), we can determine the normal contact force $F_{i}^{n}$ using the potential energy $E_{i}$ stored in the contact set. With the help of $F_{i}^{n}=\mathrm{d} P_{i}^{n} / \mathrm{d} t$, the ratio of the normal infinitesimal impulsive between different contact sets $R_{i j}^{n}$ can be obtained by using the contact stiffness and the potential energy distribution in these contact sets,

$$
R_{i j}^{n} \triangleq \frac{\mathrm{d} P_{i}^{n}}{\mathrm{~d} P_{j}^{n}}=\frac{F_{i}^{n}}{F_{j}^{n}}=\frac{\left(\alpha_{i}+1\right)^{\frac{\alpha_{i}}{\left(\alpha_{i}+1\right)}} k_{i}^{\frac{\alpha_{i}}{\left(\alpha_{i}+1\right)}} E_{i}^{\frac{\alpha_{i}}{\left(\alpha_{i}+1\right)}}}{\left(\alpha_{j}+1\right)^{\frac{\alpha_{j}}{\left(\alpha_{j}+1\right)}} k_{j}^{\frac{\alpha_{j}}{\left(\alpha_{j}+1\right)}} E_{j}^{\frac{\alpha_{j}}{\left(\alpha_{j}+1\right)}}}
$$

Supposing that all of the contact points take the same power in (26), namely, $\alpha_{i}=\alpha_{j}=\alpha$, we can simplify the Eq. (28) as

$$
R_{i j}^{n} \triangleq \frac{\mathrm{d} P_{i}^{n}}{\mathrm{~d} P_{j}^{n}}=\frac{F_{i}^{n}}{F_{j}^{n}}=\left(\frac{k_{i}}{k_{j}}\right)^{\frac{\alpha}{\alpha+1}}\left(\frac{E_{i}}{E_{j}}\right)^{\frac{\alpha}{\alpha+1}} .
$$

Generally, we select the contact point $j$ which has the maximal potential energy among all the closed contact sets, i.e. $E_{j}=\max \left\{E_{i}, i=1,2,3, \cdots, \kappa\right\}$, as the primary impact point, and define $\mathrm{d} P_{j}^{n}$ as the primary differential impulse. Hence, by using Eq. (27), we have

$$
\mathrm{d} \mathbf{P}^{n}=\mathbf{R}^{n} \mathrm{~d} P_{j}^{n}
$$

where $\mathbf{R}^{n}=\left[R_{1, j}^{n}, R_{2, j}^{n}, \cdots, R_{2 \kappa-2+\lambda, j}^{n}\right]^{T}$ is the normal distributing matrix.

For the tangential differential impulse, we introduce the Coulomb friction to calculate the ratio $R_{i j}^{\tau}$ between the tangential differential impulse $\mathrm{d} P_{i}^{\tau}$ and the primary normal impulses $\mathrm{d} P_{j}^{n}$, and the distribution of the tangential differential impulse can be rewritten as

$$
\mathrm{d} \mathbf{P}^{\tau}=\mathbf{R}^{\tau} \mathrm{d} P_{j}^{n}
$$

where $\mathbf{R}^{\tau}=\left[R_{1, j}^{\tau}, R_{2, j}^{\tau}, \cdots, R_{2 \kappa-2+\lambda, j}^{\tau}\right]^{T}$ is the tangential distributing matrix. Now, applying Eqs. (29) and (30) to Eq. (21), it gives

$$
\mathbf{M d} \dot{\mathbf{q}}=\left(\mathbf{W R}^{n}+\mathbf{N R}^{\tau}\right) \mathrm{d} p_{j}^{n}
$$

For starting the simulation of the domino system under the assumption that there is no pre-press potential energy stored at the contact sets, the relationship between the initial normal infinitesimal impulse distribution and the initial velocity

$$
\frac{d P_{i}^{n}}{d P_{j}^{n}}=\left(\frac{k_{i}}{k_{j}}\right)^{\alpha+1}\left(\frac{-\dot{\delta}_{i}(0)}{-\dot{\delta}_{j}(0)}\right)^{\frac{\alpha}{\alpha+1}}
$$

should be utilized for initial calculation [22, 23].

In general, we select the contact point $j$ which has the maximal normal relative velocity among all the closed contact sets, i.e. $\left|\dot{\delta}_{j}(0)\right|=\max \left\{\left|\dot{\delta}_{i}(0)\right|, i=1,2,3, \cdots \kappa\right\}$, as the primary impact point. Then the ratio matrices $\mathbf{R}^{n}$ and $\mathbf{R}^{\tau}$ will be dynamically updated based on the evolution of the 
potential energy. The multi-impact process will finish once all the potential energy stored in the contact sets are released, i.e. $E_{i}=0$, where $i=1,2, \ldots, \kappa$. Here, it is worth noting that the potential energy at the contact points might not disappear simultaneously.

\section{Numerical simulation and model validation}

This section presents the numerical results of the domino model studied in Section 2. Comparisons with the experimental results shown in [1, 18] will be given to validate the numerical model. By setting the physical parameters satisfying the assumptions introduced by Stronge in [1], we will show that Stronge's theoretical model indeed sheds essential insights on the mechanism of domino wave. Similar comparisons between the results obtained from our numerical model and the theoretical model by van Leeuwen [13] and Fujii et. al [21] will also be given. Furthermore, how the physical parameters affect the domino wave will be investigated through comprehensive numerical simulations.

\subsection{Comparison of numerical and experimental results}

For regularly spaced dominoes with identical slender rectangular blocks, the motion state of each block during the toppling process is irrelevant to the mass of the block. Therefore, we can set $m=1$ in our simulations without loss of generality. Following Stronge, we introduce a natural speed scale $\bar{v}=\sqrt{g h}$ and a natural time scale $\bar{t}=\sqrt{h / g}$ to present the numerical results in a dimensionless form.

Stronge [1, 18] performed experiments by using three kinds of blocks. They are referred to as domino $\mathrm{X}, \mathrm{T}$ and $\mathrm{P}$ in this paper. The physical parameters used in our simulations are shown in Table 1, in which the underlined data were extracted from [1, 18]. Other parameters, such as the coefficients of restitution and friction, the contact stiffness, for the domino-domino and domino-ground interactions, are estimated according to the physical properties of the block and ground materials. It is worth noting that only the ratios of the contact stiffness between different contact points are needed in our numerical model. In addition, the number of discrete points for the line contact of the $\kappa$ th block was set to $\lambda=9$, and the power for the constitutive equation in Eq. (25) was set to $\alpha=1.5$. Numerical integration was implemented by using the improved Euler method with a time step $\Delta t=$ $1 \times 10^{-4} s$. The impulse step in solving impact dynamics was set to $\Delta p_{j}=10^{-4} P_{0}$, where $P_{0}$ is the amplitude of the linear momentum of the system just before the impact.

Our first comparison between numerical and experimental results is carried out for a domino system composed of Domino X with an array spacing of $s / h=0.18$. Stronge provided the experimental data for the intercollision period during toppling of dominoes (see Figure 4 in [18]). Accordingly, we define a dimensionless propagation speed $\hat{v}$ as

$$
\hat{v}=\frac{s+d}{t_{i} \bar{v}}
$$

where $t_{i}$ is the time interval for the $i$ th block from the start of toppling to the collision with the $(i+1)$ th block.
The work [18] provided two sets of experimental data for the toppling of dominoes triggered by two different initial speeds of the first block. Nevertheless, the author did not mention the concrete values of the initial speeds. In order to have the best match with the experimental results in [18], we assigned the first block to initially rotate around its bottom corner with dimensionless angular velocities, $\hat{\dot{\theta}}_{1}(t=$ $0)=1.8$ and 0.5 , where $\hat{\dot{\theta}}_{i}=\dot{\theta}_{i} \bar{t}$, for fast and slow toppling, respectively. Fig. 3 presents the dimensionless propagation speed $\hat{v}$ as a function of the number of domino blocks $n$, where simulation and experimental results are shown in red and blue markers, respectively.

As can be seen from the Fig. 3, our numerical results are in good agreement with the experimental results. As expected, the propagation speed tends to a stable value, i.e. the natural speed, which is independent of the initial toppling angular speed. Nevertheless, the value of the natural speed is not fixed, yet it fluctuates with the wave propagation. We will explain this phenomenon in detail in the later subsection.

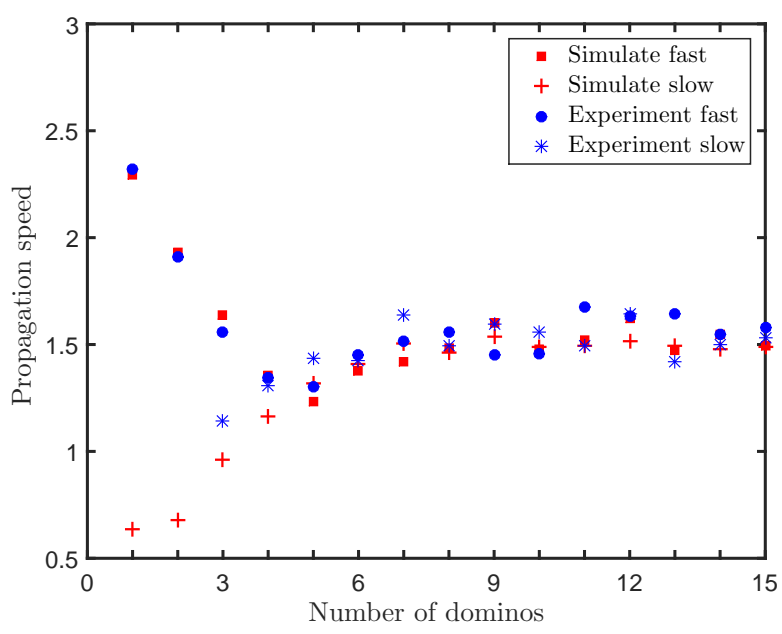

Fig. 3. (Colour online) Dimensionless propagation speed $\hat{v}$ varies as a function of the number of dominoes $n$ for different initial toppling speeds. Parameters of Domino X in Table 1 were used. To compare with experimental results, our calculations were carried out using a fast initial dimensionless angular speed at 1.8 and a slow one at 0.5 . Simulation results for fast and slow initial toppling angular speeds are marked by $\square$ and + , and the experimental results obtained from 18 for fast and slow initial toppling angular speeds are marked by $\bullet$ and $*$, respectively.

The dimensionless natural speed, denoted by $\hat{v}_{n a}$ in this paper for a stable domino wave, is independent of the initial perturbation exerted on the first domino, and is predominately governed by the domino spacing, the sliding friction and the coefficient of restitution of the domino-domino contact. In [18], Stronge and Shu presented the experimental results for the speed varying with the domino spacing. The parameters of Domino $\mathrm{T}$ and $\mathrm{P}$ given in Table 1 were adopted in our simulations. After the collision wavefront has passed through the first 6 blocks, the dimensionless speeds, which can be calculated using Eq.(33), settles down to a small but 
Table 1. Geometric and material parameters of the domino systems.

\begin{tabular}{|c|c|c|c|}
\hline & Domino X & Domino $\mathrm{T}$ & Domino P \\
\hline Material & Plastic & Tufnol & Perspex \\
\hline \multicolumn{4}{|c|}{ Geometry (m) } \\
\hline Height $h$ & $\underline{4.18 \times 10^{-2}}$ & $\underline{8.00 \times 10^{-2}}$ & $\underline{8.00 \times 10^{-2}}$ \\
\hline Thickness $d$ & $\underline{7.58 \times 10^{-3}}$ & $\underline{9.60 \times 10^{-3}}$ & $\underline{9.90 \times 10^{-3}}$ \\
\hline \multicolumn{4}{|c|}{ Slip coefficient of friction ${ }^{\star}$} \\
\hline Domino $\mu_{1}$ & $\underline{0.17}$ & $\underline{0.15}$ & $\underline{0.25}$ \\
\hline Ground $\mu_{2}$ & 0.25 & 0.20 & 0.30 \\
\hline \multicolumn{4}{|c|}{ Stick coefficient of friction } \\
\hline Domino $\mu_{1}^{s}$ & 0.20 & 0.18 & 0.30 \\
\hline Ground $\mu_{2}^{s}$ & 0.30 & 0.25 & 0.36 \\
\hline \multicolumn{4}{|c|}{ Coefficients of restitution } \\
\hline Domino $e_{1}$ & $\underline{0.85}$ & $\underline{0.62}$ & $\underline{0.55}$ \\
\hline Ground $e_{2}$ & 0.50 & 0.50 & 0.50 \\
\hline \multicolumn{4}{|c|}{ Contact stiffness $\left(\mathrm{N} / \mathrm{m}^{3 / 2}\right)$} \\
\hline Domino $k_{1}$ & $2.30 \times 10^{8}$ & $2.30 \times 10^{8}$ & $2.30 \times 10^{8}$ \\
\hline Ground $k_{2}$ & $6.90 \times 10^{8}$ & $6.90 \times 10^{8}$ & $6.90 \times 10^{8}$ \\
\hline
\end{tabular}

* Domino X corresponds to the domino block in [18], and Domino T and P represent the tufnol and the perspex domino blocks in [1], respectively. The physical parameters between two blocks are labelled by combining with word the "Domino", while the ones between a block and the ground are stated by combining with the word "Ground". Underlined data were extracted from [18]1]. $\star$ The coefficient of sliding friction in impact is smaller than the one in contact due to vibration agitated by impact [29 30]. In simulation, the values of the coefficient of sliding friction in impact are half of the ones listed in the table.

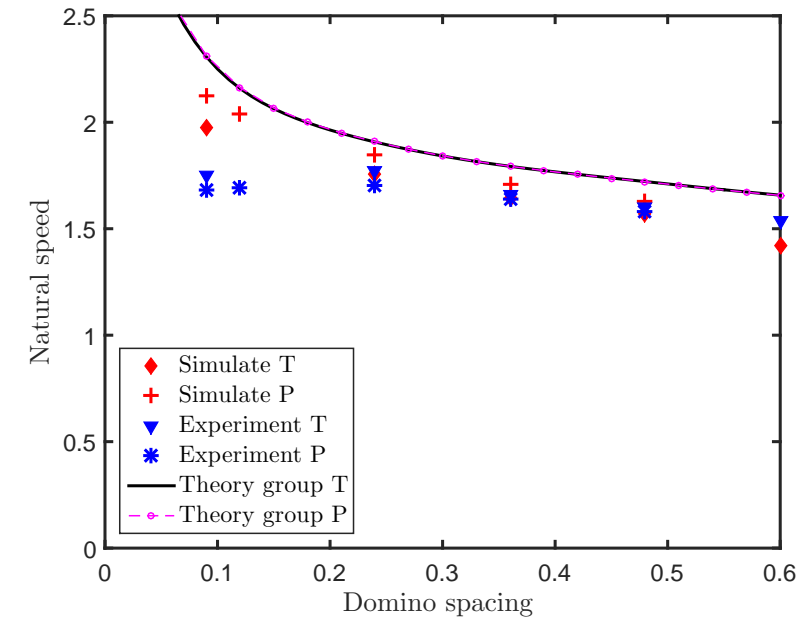

Fig. 4. (Colour online) Dimensionless speed $\hat{v}_{n a}$ varies as a function of the domino spacing $s / h$ for Domino $\mathrm{T}$ and $\mathrm{P}$ in Table 1 Simulation results using the proposed domino model are marked by $\checkmark$ and + , and the experimental results obtained from [1] are given by $\boldsymbol{\nabla}$ and $*$. Theoretical solution for Domino $\mathrm{T}$ and $\mathrm{P}$ calculated using the Stronge's cooperative group model is denoted by the solid and dot-dash line, respectively.

regular variation around a constant. The values of $\hat{v}_{n a}$ for each spacing are presented in Fig. 4. As can be seen from the figure, the numerical results obtained by our proposed model have a good agreement with the experimental results in [1] for both tufnol and perspex domino blocks.

\subsection{Comparison of numerical and theoretical results}

For capturing the essential features of the domino dynamics, Stronge [18] proposed a simple single collision model by introducing the following assumptions:

(1) The toppling process initiates a sequence of collisions where each toppling block knocks over its neighbor;

(2) The toppling block before collision rotates about an edge without slippage on the ground.

(3) The collision between two blocks is treated as a frictional impact.

Based on the above assumptions, a simple relationship for the natural speed of the steady collision propagation can be obtained, which is a function depending on the domino spacing, sliding friction and the coefficient of restitution in the domino-domino contact.

The main problem concerned by the single collision model is that the coupling of interaction in a group of neighbouring blocks is neglected completely. In order to capture the united action of a cooperative group, Stronge [1] developed a cooperative group model by introducing the following assumptions:

(i) there is an indefinitely large number of toppling dominoes behind the collision wavefront;

(ii) each domino behind the wavefront leans forward against a neighbour;

(iii) during toppling, friction at the sliding contact between dominoes is negligible; 
(iv) after a collision, colliding blocks remain in sliding contact;

(v) no slippage occurs at the contact between the block and the ground.

The cooperative group model gives a prediction for the natural speed irrelevant to domino's material parameters, while only depending on domino's geometric parameters and spacing of domino's array. In terms of the parameters of Domino T given in Table 1, the theoretical solutions for the dimensionless natural speed, calculated using the Stronge's cooperative group model and the single collision model, are shown in solid and dot-dash lines in Fig. 5, respectively. Both the theoretical solutions take a large discrepancy in comparison with the experimental results.

Although the ideal scenario described by the assumptions of the Stonge's theoretical model is hardly replicated by experiments, it can be simulated by setting ideal values to the material parameters in numerical simulations. For instance, to prevent block slipping and rebounding on the ground in toppling process, we can set a large coefficient of friction $\left(\mu_{2}\right)$ and the coefficient of restitution $e_{2}=0$ for the contact between dominoes and the ground. In order to make the colliding blocks remain in frictionless sliding contact after collisions, we can assign $\mu_{1}=0$ and $e_{1}=0$ for dominodomino contact in our numerical model. Fig. 5 shows that the numerical results obtained from our model, in which the model parameters enable the Stronge's assumptions to be satisfied, can agree with the theoretical results of the Stronge's cooperative group model. This figure also shows that the theoretical results obtained from the single impact model deviate far from our numerical results. Obviously the cooperative interaction between multiple dominoes is inevitable and it plays a significant role of affecting the wave propagation. Meanwhile, the cooperative group model under the Stronge's assumptions is just a special case of our proposed model.

By adopting the same assumptions in Stronge's cooperative group model except item (iii), Fujii et. al [21] and Leeuwen [13] have developed similar models taking into account the friction effects between dominoes on the wave propagation, and established iterative formulations for computing the natural speed of the wave propagation. This model is termed as the extended group model. Fig. 5 shows the extended group model can give a good prediction when domino spacing is not sufficiently large. For a large domino spacing, however, a relative large error exists between the results of the extended group model and our numerical model. This suggests that, if domino spacing is sufficiently large, the primary collision of the wavefront may result in separation or stick-slip motion between toppling dominoes, leading to errors between the theoretical predictions of the cooperative group model and the numerical results obtained from our numerical model, which can help us to capture the details of motion of each domino during the wave propagation.

\subsection{Details of the domino waveform}

Exposure for the details of the domino waveform can help us to understand the intrinsic property of the wave

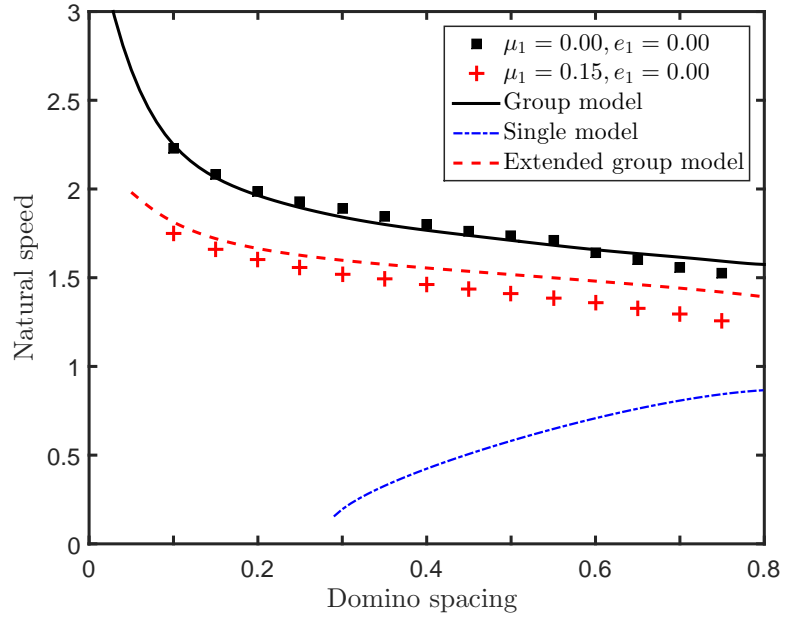

Fig. 5. (Colour online) Dimensionless natural speed $\hat{v}_{n a}$ varies as a function of the domino spacing $s / h$ for Domino T in Table 1 In all of the simulations, the material parameters between domino and ground are always set to $e_{2}=0$ and $\mu_{2}=0.9$. Numerical results obtained by using different material parameters between dominos are plotted as follows: $\mathbf{\square}\left(e_{1}=0, \mu_{1}=0\right)$; and $+\left(\mu_{1}=0.15, e_{1}=\right.$ $0)$. Theoretical solutions calculated by the cooperative group model, the single collision model and the extended group model are denoted by solid line, dot-dash line and dash line, respectively.

propagation, and to unclose the dependence of the waveform profile on the physical parameters of the dominoes. Fig. 6 shows two sets of snapshots of the toppling domino array with the spacing of $s / h=0.52$ obtained from numerical simulations by using two sets of model parameters. The first set corresponds to the parameters of Domino $\mathrm{X}$ given in Table 1 while the second set modifies part of model parameters of Domino $\mathrm{X}$ as follows: $\mu_{1}=0, e_{1}=0, \mu_{2}=$ 0.9 , and $e_{2}=0$. The second set of the model parameters makes Domino $\mathrm{X}$ free of sliding friction and restitution in the domino-domino contact. In this case the assumption of the Stronge's cooperative group model is satisfied, and a complete solitary propagation wave can be formed in the toppling process. Fig. 6 shows the snapshots captured from our numerical simulations under the two sets of the model parameters by using the same initial condition. It can be seen that the rebounds between two colliding dominoes clearly exist in Fig. 6a, but in Fig. 6b, colliding blocks remain in sliding contact during toppling.

Fig. 7 shows the dimensionless angular velocities $\hat{\dot{\theta}}$ of the 7th-10th dominoes obtained from simulations under the two sets of parameters. Due to the blocks starting their motion at the different moments, the moment $t=0$ in Fig. 7 is defined as the moment when the relevant domino forms a contact with its neighbor. As can be seen from this figure, each domino experiences several impacts reflected by the sharp change of velocity, and all the blocks terminate at a stationary state. Fig.7 (7) shows the results obtained from the first set of the model parameters. Clearly the dominoes have anti-clockwise rotation, i.e. negative angular velocity, when collision with neighboring block occurs. The numerical results for the second set of the model parameters shows that dominoes only have clockwise rotation as observed 

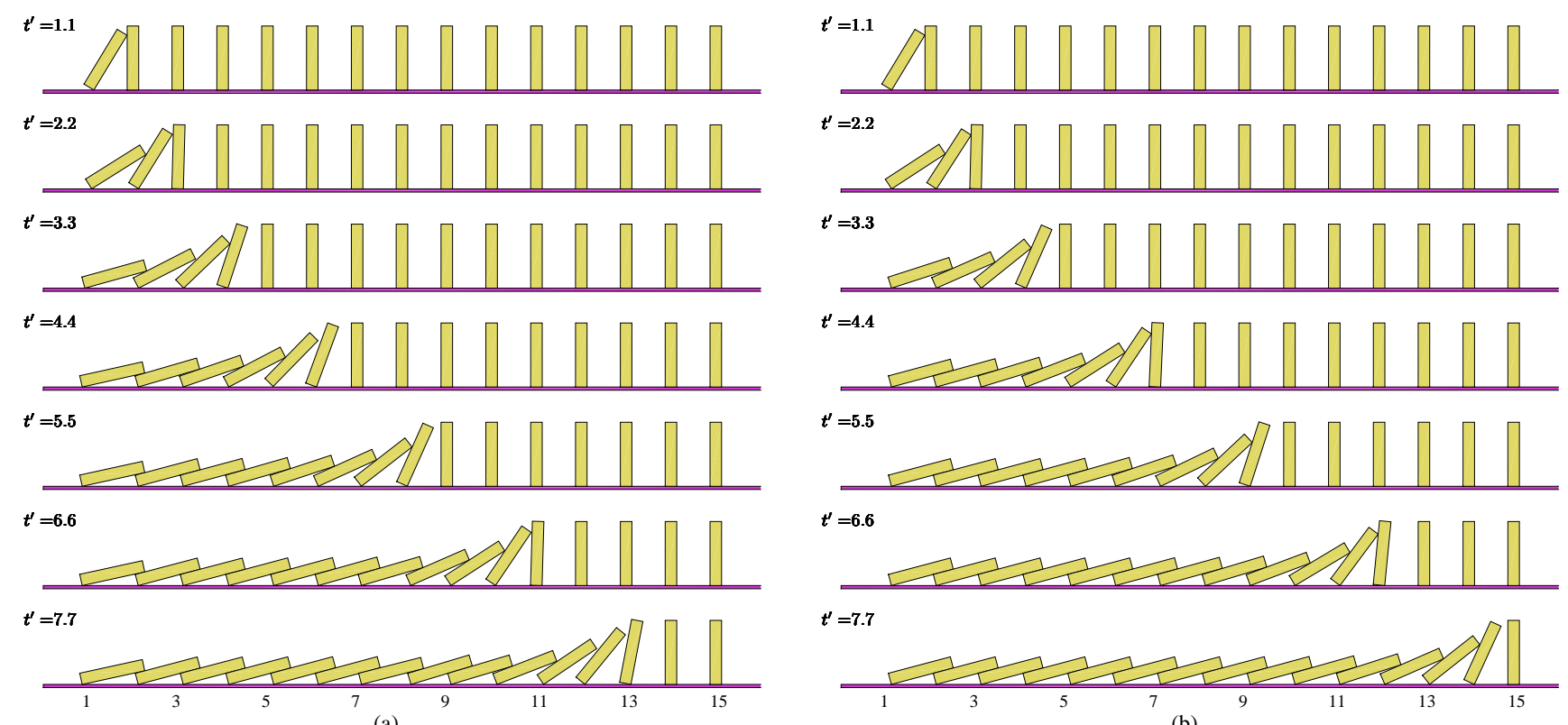

(a)

(b)

Fig. 6. Snapshots of the toppling of a domino array separated by a spacing of $s / h=0.52$. Simulations were performed by using the parameters of Domino X given in Table 1 for (a) $\mu_{1}=0.17, e_{1}=0.85, \mu_{2}=0.25$ and $e_{2}=0.5$; (b) $\mu_{1}=0, e_{1}=0, \mu_{2}=0.9$ and $e_{2}=0$.

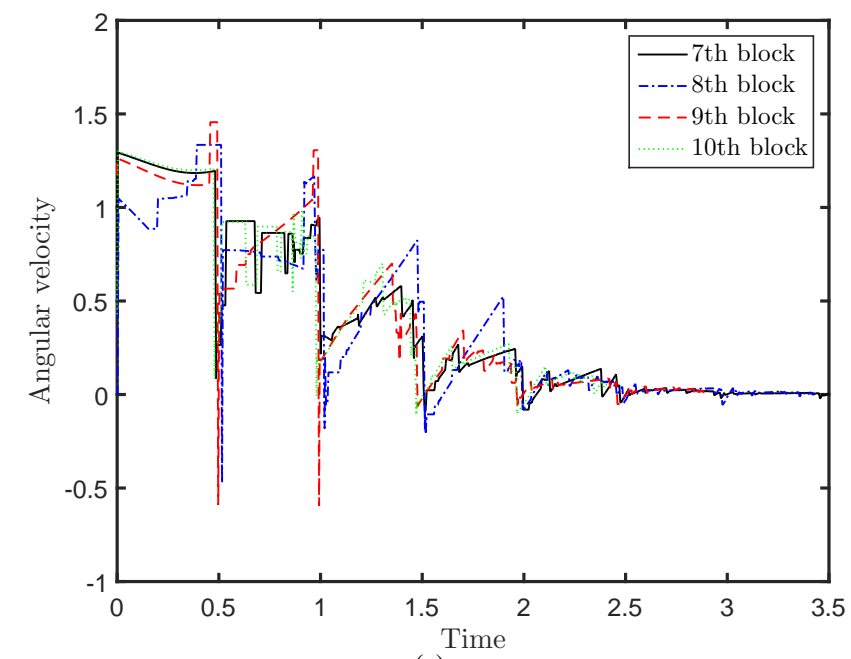

(a)

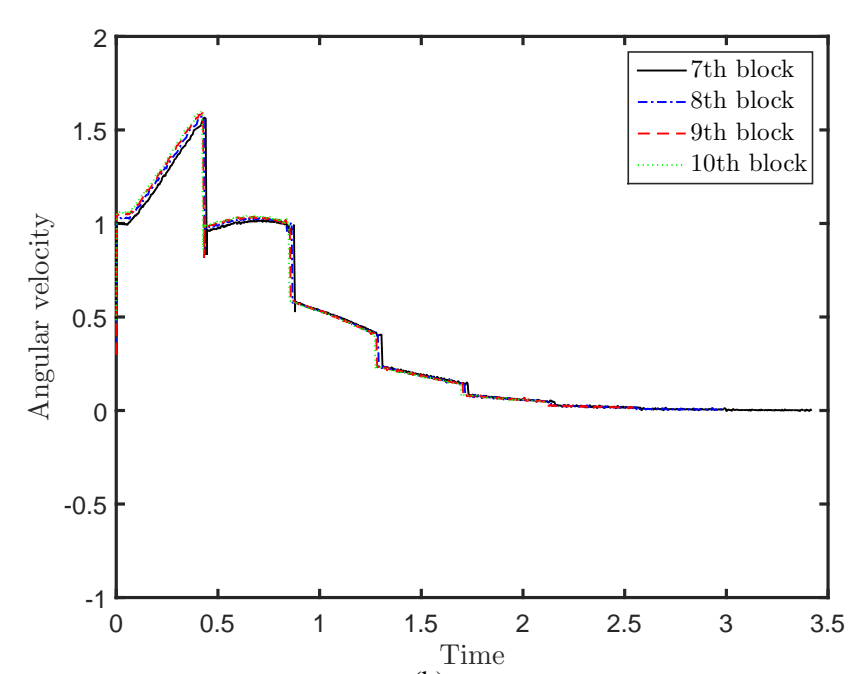

(b)

Fig. 7. (Colour online) Dimensionless angular velocity $\hat{\dot{\theta}}_{i}$ of 7th, 8th, 9th, and 10th block as a function of dimensionless time $t / \bar{t}$ obtained from simulations by using the parameters of Domino X given in Table 1 for (a) $\mu_{1}=0.17, e_{1}=0.85, \mu_{2}=0.25$ and $e_{2}=0.5$; (b) $\mu_{1}=0$, $e_{1}=0, \mu_{2}=0.9$ and $e_{2}=0$. Time $t=0$ is defined as the moment when the domino forms a contact with its left neighbor.

from Fig. 7b). This implies that there is no rebounding between blocks when collision occurs. Another observation is that the velocity waveform is exactly transmitted from the 7 th domino to the 10th one for the simulation under the second set of model parameters. Simulations under the first set of model parameters reveal that the waveform becomes irregular due to multiple and repetitious impacts. This explains the reason why some fluctuations around its natural speed have been observed in Fig. 3 .

A further comparison for the numerical results obtained from the two sets of model parameters under various initial toppling velocities is shown in Fig. 8, where the temporal evolution of propagation speed with the number of dominoes is given. Fig. 8 a) presents the numerical results obtained from the first set of model parameters, and it can be seen that the propagation speeds under different initial conditions asymptotically approaches a natural speed whose value fluctuates around an average constant. The fluctuation is due to the multiple and repetitious impacts between dominoes, which can be seen from the serration of the curve of angular velocity in Fig. 7 (a). When simulations were performed under the second set of model parameters, those events cannot occur in the process of the wave propagation, such that the propagation speeds can converge to a constant intrinsic speed, as observed from Fig. 8(b). Fujii et. al [21] used their extended group model to study the wave propagation and obtained similar results as shown in Fig. 8b. 


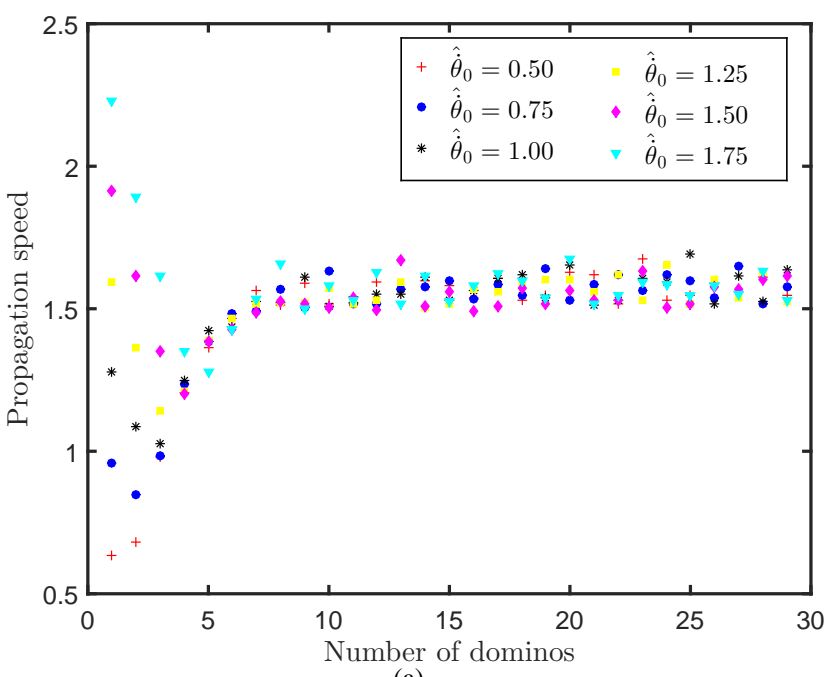

(a)

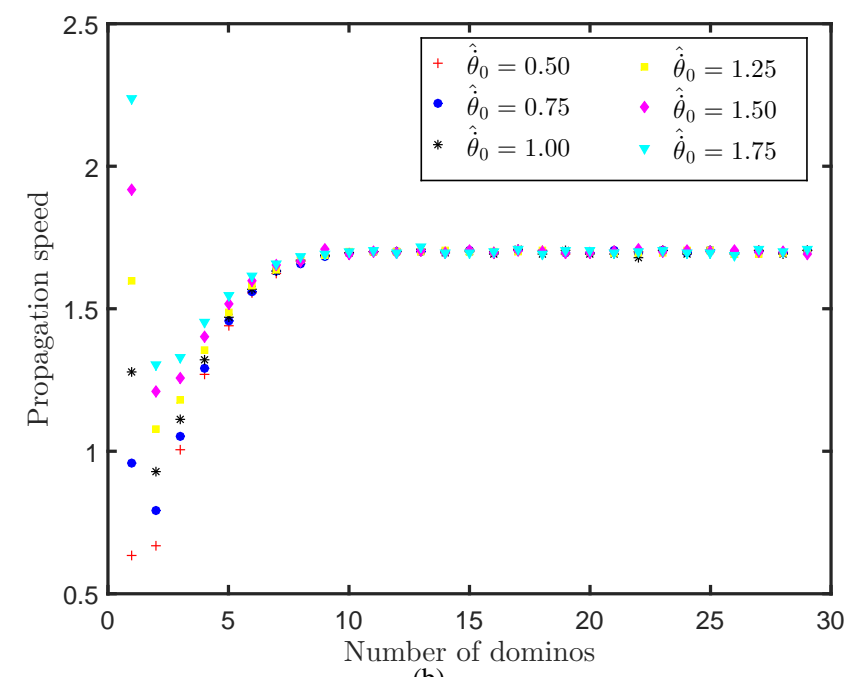

(b)

Fig. 8. (Colour online) Dimensionless propagation speed $\hat{v}$ varies as a function of the number of dominoes $n$ calculated for parameters of Domino X in Table 1for (a) $\mu_{1}=0.17, e_{1}=0.85, \mu_{2}=0.25$ and $e_{2}=0.5$; (b) $\mu_{1}=0, e_{1}=0, \mu_{2}=0.9$ and $e_{2}=0$ under different initial toppling speeds cases.

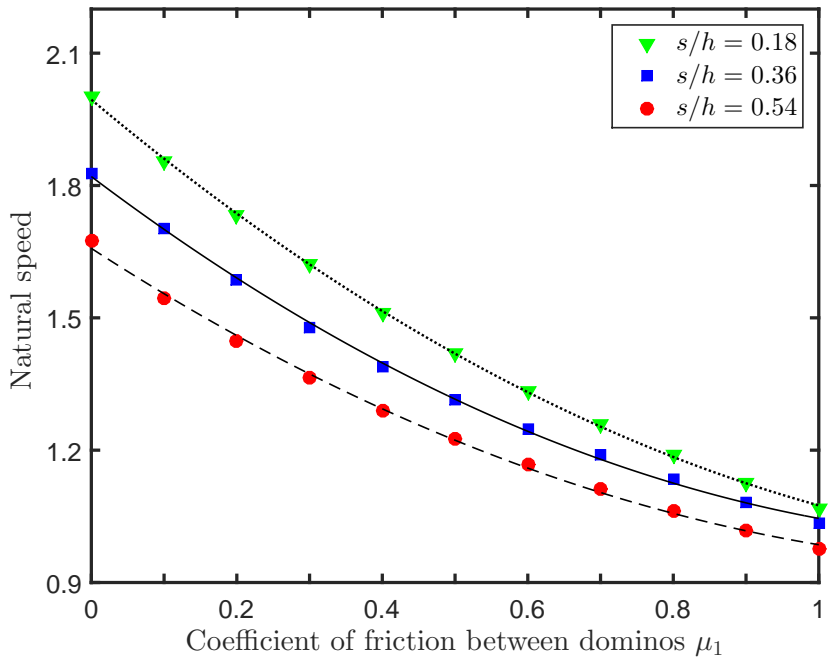

(a)

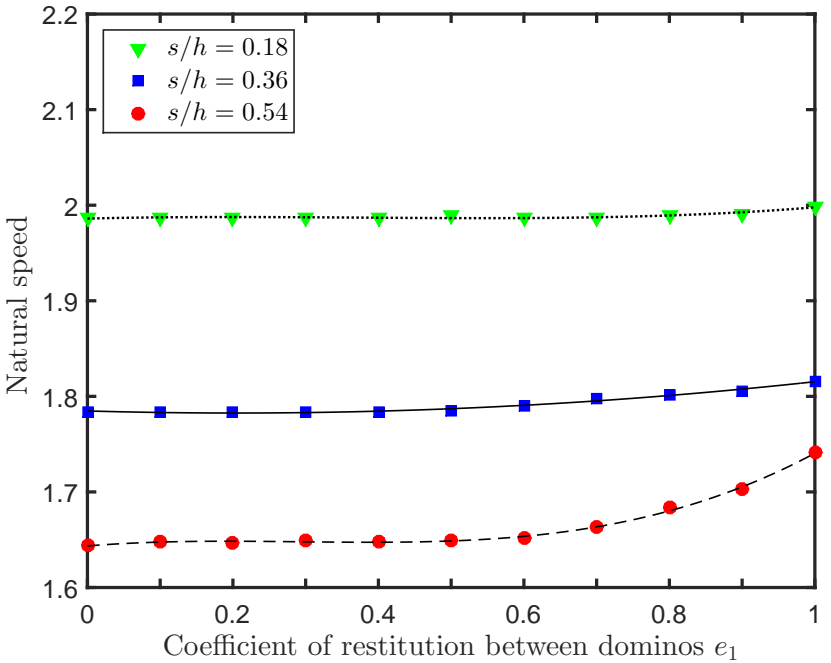

(b)

Fig. 9. (Colour online) Dimensionless natural speed $\hat{v}_{n a}$ as a function of (a) coefficient of friction $\mu_{1}$ calculated for $d / h=0.18, \mu_{2}=0.9$, $e_{1}=0$ and $e_{2}=0$; (b) coefficient of restitution $e_{1}$ calculated for $d / h=0.18, \mu_{2}=0.9, e_{2}=0$ and $\mu_{1}=0$; for different relative spacing $s / d$.

\subsection{Influence of physical parameters on natural speed}

In this part, influence of the physical parameters, $\mu_{1}$, $e_{1}, \mu_{2}$, and $e_{2}$ on the natural speed of the domino system is investigated by using the numerical model proposed in this paper. The geometric parameters of Domino $\mathrm{X}$ given in Table 1 are used in the numerical investigations. For each set of model parameters, we carry out numerical simulations under three different spacings.

Fig. 9 and Fig. 10 show how the sliding friction and the coefficient of restitution in domino-domino contact and the ones in domino-ground contact affect the natural speed of the collision wave. Fig.9(a) and Fig. 10(a) demonstrate that the speed decreases as either of the coefficients of friction $\mu_{1}$ or $\mu_{2}$ increases, since increased friction may cause more energy dissipation. In particular, the speed will dramatically decrease with the increase of the friction between dominoes, since the friction is the primary mechanism responsible for speed/energy propagation. For the coefficients of restitution $e_{1}$ and $e_{2}$, their influences on the natural speed are not such great, but as the coefficient of restitution between dominoes $e_{1}$ is large enough, an obvious increase of the natural speed can be observed in Fig. 9(b). This is due to the fact that as the coefficient of restitution $e_{1}$ increases, less energy is dissipated during elastic impact. Another reason for the speed increasing with $e_{1}$ comes from the rebound motion of dominoes triggered by collisions. Obviously the rebound motion will be enhanced by $e_{1}$, leading to separation between dominoes, and finally affecting the propagation speed. The spacing between dominoes, as shown in all of these figures, is a significant influencing factor of the natural speed. 


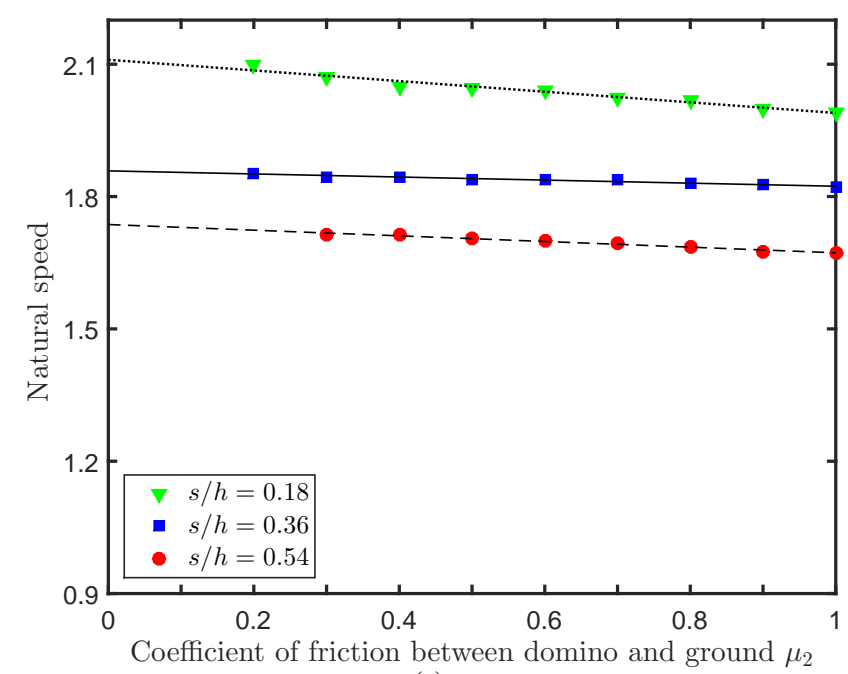

(a)

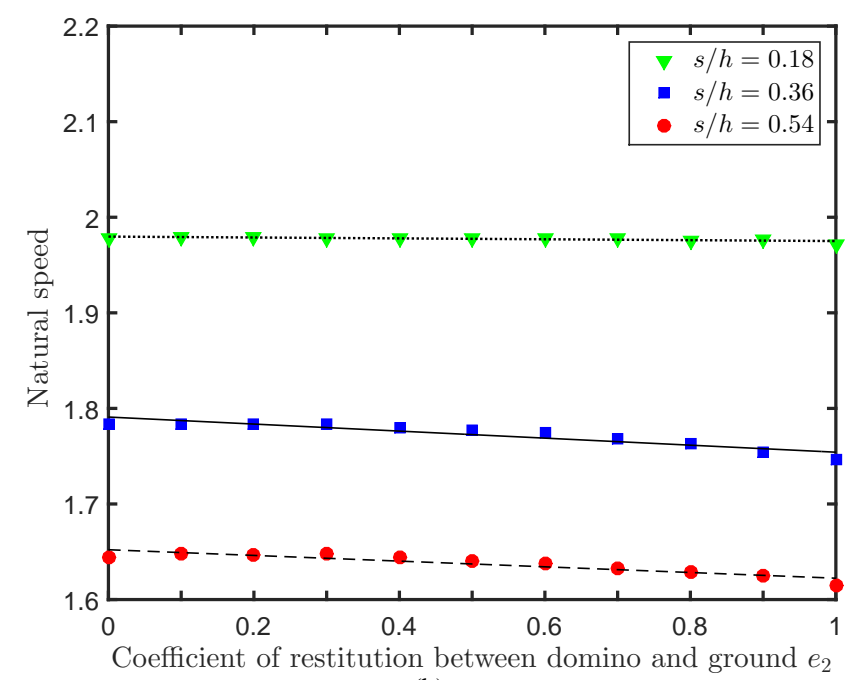

(b)

Fig. 10. (Colour online) Dimensionless natural speed $\hat{v}_{n a}$ as a function of (a) coefficient of friction $\mu_{2}$ calculated for $d / h=0.18, \mu_{1}=0$, $e_{1}=0$ and $e_{2}=0$; (b) coefficient of restitution $e_{2}$ between domino and ground calculated for $d / h=0.18, e_{1}=0, \mu_{2}=0.9$ and $\mu_{1}=0$; for different relative spacing $s / d$.

\section{Conclusions}

A new comprehensive model for toppling dynamics of regularly spaced dominoes in an array was studied in this paper. The model has removed the hypotheses of the cooperative group model proposed by Stronge [1], and the extended cooperative group model proposed by Fujii et. al [21] and van Leeuwen [13]. Stronge's model completely neglects the effects of friction and restitution between dominoes on wave propagation, leading to the dependency of natural speed only on domino's geometry and arrangement of the domino's array. The extended cooperative group model modifies Stronge's model by considering friction effects on wave speed, while it cannot reflect the restitution effects of domino collisions, thus leading to errors for wave speed prediction. The numerical model proposed in this paper can reflect the coupling effects among simultaneous contact points, and precisely detail the motion of each domino.

Our proposed model was validated using the experimental results studied in [1, 18]. Some further insights into the dynamics of falling domino were also taken by investigating the collision propagation under different model parameters. For a domino array with a small spacing, it has been found that the extended cooperative group model developed in [13. 21] can give a good prediction for wave speed if no attention is paid on the details of the toppling dynamics. For the cases of the domino system with a large spacing, the effect of separation or stick-slip motion in toppling dominoes on wave speed cannot be ignorable. Therefore, a relative large error may exist when the cooperative group models reported in [1, 13, 21] are used. Actually, the fluctuation in the waveform of domino's propagation speed is caused by the irregular multiple impacts between colliding dominoes, in which there are frequent switches among separation and closure of the contacts between neighboring dominoes. To some extent, this fluctuation affects the wave speed. In addition, our numerical results suggest that the wave speed of toppling dominoes decreases as the coefficient of friction increases. On the other hand, influences of the coefficients of restitution on the speed are not so obvious.

In summary, the domino system exhibits interesting dynamical phenomena resulted from the combined actions of a group of dominoes. Our proposed model can expose detailed information of each domino's motion by properly taking into account the influence of the restitution and friction between dominoes and the ground on domino propagation. This model is more realistic and has not been considered in the previous studies. It is worth noting that contact and impact widely exist in mechanical machnies, therefore, this study also has significant implications for the dynamics of mechanical systems subject to complicated contact and impact interactions.

\section{Acknowledgment}

This work was performed under the support of the National Natural Science Foundation of China (NSFC:11472011,11702002). The authors would like to specially thank Professor Jianhua Xie for providing detailed and valuable idea.

\section{References}

[1] Stronge, W. J., and Shu, D., 1988. "The domino effect: successive destabilization by cooperative neighbours". Proceedings of the Royal Society A: Mathematical, Physical and Engineering Science, 418(1854), pp. 155-163.

[2] Olson, E. L., and Allen, R. M., 2005. "The deterministic nature of earthquake rupture". Nature, 438(7065), pp. 212-215.

[3] Yuan, R. M., Xu, X. W., Chen, G. H., Tan, X. B., Klinger, Y., and Xing, H. L., 2010. "Ejection landslide at northern terminus of beichuan rupture triggered by the $2008 \mathrm{mw} 7.9$ wenchuan earthquake". Bulletin 
of the Seismological Society of America, 100(5B), pp. 2689-2699.

[4] Gonze, D., and Goldbeter, A., 2001. "A model for a network of phosphorylation-dephosphorylation cycles displaying the dynamics of dominoes and clocks". Journal of theoretical biology, 210(2), pp. 167-186.

[5] Stevens, W. M., 2012. "Computing with planar toppling domino arrangements". Natural Computing, 11(4), pp. 665-672.

[6] Chang, T., and Guo, Z., 2010. "Temperature-induced reversible dominoes in carbon nanotubes". Nano letters, 10(9), pp. 3490-3493.

[7] Abdolhamidzadeh, B., Abbasi, T., Rashtchian, D., and Abbasi, S. A., 2011. "Domino effect in processindustry accidents-an inventory of past events and identification of some patterns". Journal of Loss Prevention in the Process Industries, 24(5), pp. 575593.

[8] Nakabayashi, S., Sugiyama, N., Yagi, I., and Uosaki, K., 1996. "Dissociative adsorption dynamics of formaldehyde on a platinum electrode surface; onedimensional domino?". Chemical physics, 205(1-2), pp. 269-275.

[9] Daykin, D., 1971. "Falling dominoes". SIAM Review, 13(4), p. 569.

[10] Maddox, J., 1987. "The domino effect explained". Nature, 325, p. 191.

[11] Wagon, S., Pontarelli, A., Briggs, W., and Becker, S., 2005. "The dynamics of falling dominoes". The UMAP Journal, 26(1), pp. 35-47.

[12] Pelesko, J. A., 2005. Falling dominoes. at http://www . math. udel . edu/ pelesko/Teaching PDF file.

[13] Van Leeuwen, J. M. J., 2010. "The domino effect". American Journal of Physics, 78(7), pp. 721-727.

[14] Lu, G., Third, J. R., and Müller, C. R., 2014. "Effect of particle shape on domino wave propagation: a perspective from $3 \mathrm{~d}$, anisotropic discrete element simulations". Granular matter, 16(1), pp. 107-114.

[15] McLachlan, B., Beaupre, G., Cox, A., and Gore, L., 1983. "Falling dominoes (de daykin)". SIAM Review, 25(3), p. 403.

[16] Bert, C. W., 1986. "Falling dominoes". SIAM Review, 28(2), pp. 219-224.

[17] Efthimiou, C. J., and Johnson, M. D., 2007. "Domino waves". SIAM review, 49(1), pp. 111-120.

[18] Stronge, W. J., 1987. "The domino effect: a wave of destabilizing collisions in a periodic array". Proceedings of the Royal Society A: Mathematical, Physical and Engineering Science, 409(1836), pp. 199-208.

[19] Larham, R., 2008. "Validation of a model of the domino effect?". arXiv preprint arXiv:0803.2898.

[20] Shaw, D., 1978. "Mechanics of a chain of dominoes". American Journal of Physics, 46(6), pp. 640-642.

[21] Fujii, F., Inoue, Y., and Nitta, T., 2012. "Modeling the domino wave propagation in contact mechanics". Transactions of The Japan Society of Mechanical Engineers Series C, 78(788), pp. 1133-1142.

[22] Liu, C., Zhao, Z., and Brogliato, B., 2008. "Frictionless multiple impacts in multibody systems. i. theoretical framework". Proceedings of the Royal Society of London A: Mathematical, Physical and Engineering Sciences, 464(2100), pp. 3193-3211.

[23] Liu, C., Zhao, Z., and Brogliato, B., 2009. "Frictionless multiple impacts in multibody systems. ii. numerical algorithm and simulation results". Proceedings of the Royal Society of London A: Mathematical, Physical and Engineering Sciences, 465(2101), pp. 1-23.

[24] Zhao, Z., and Liu, C., 2016. "Contact constraints and dynamical equations in lagrangian systems". Multibody System Dynamics, 38(1), pp. 77-99.

[25] Wang, J., Liu, C., and Zhao, Z., 2014. "Nonsmooth dynamics of a $3 \mathrm{~d}$ rigid body on a vibrating plate". Multibody System Dynamics, 32(2), pp. 217-239.

[26] Liu, C., Zhang, H., Zhao, Z., and Brogliato, B., 2013. "Impact-contact dynamics in a disc-ball system". In Proc. R. Soc. A, Vol. 469, The Royal Society, p. 20120741.

[27] Brogliato, B., and Brogliato, B., 1999. Nonsmooth mechanics. Springer.

[28] Jean, M., 1995. "Frictional contact in collections of rigid or deformable bodies: numerical simulation of geomaterial motions". Studies in Applied Mechanics, 42, pp. 463-486.

[29] Lun, C., and Bent, A., 1993. "Computer simulation of simple shear flow of inelastic, frictional spheres". Powders and Grains, 93, pp. 301-306.

[30] Brogliato, B., Zhang, H., and Liu, C., 2012. "Analysis of a generalized kinematic impact law for multibodymulticontact systems, with application to the planar rocking block and chains of balls". Multibody System Dynamics, 27(3), pp. 351-382. 